\title{
Near-Field Direct Antenna Modulation
}

\author{
Aydin Babakhani, David B. Rutledge, \\ and Ali Hajimiri
}

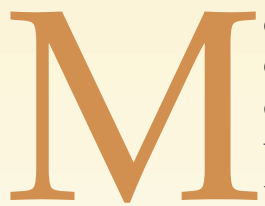

odern silicon-based technology processes have opened a plethora of opportunities for designing highly integrated millimeterwave systems by providing transistors with cutoff frequency, $f_{T}$, of more than $200 \mathrm{GHz}$ [1]-[5]. At millimeter-wave frequencies, the wavelengths are comparable to the die size, and this inspires the integration of the radiating elements and active circuit components on a single silicon die. Although integration of millimeter-wave systems on a silicon substrate lowers the cost and improves reliability, there are several challenges that must be addressed appropriately [1], [2]. Because of constraints imposed by the fabrication of active components, the substrate resistivity of the silicon substrate has to be very small $(\sim 1-10 \Omega \mathrm{cm})$. This low resistivity causes energy loss into the substrate and lowers the quality factor $Q$ of unshielded on-chip passive components such as inductors, transmission lines, and antennas and hence results in the degradation of power efficiency and noise performance. The finite conductivity of metal structures causes further energy loss in integrated systems. Since the skin depth becomes very small at mil-

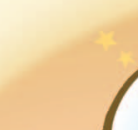
limeter-wave frequencies (e.g., the skin depth of copper at $60 \mathrm{GHz}$ is approximately $300 \mathrm{~nm}$ ), the ohmic loss in metal structures significantly increases, degrading the performance of passive devices.

As transistors become smaller and faster, their breakdown voltages become very low, introducing a serious challenge to power generation in integrated systems. Rather than implement a single amplifying block that requires a high voltage swing, it is more promising to use a parallel structure of multiple amplifying elements with a smaller swing and combine the output power.

Aydin Babakhani, David B. Rutledge, and Ali Hajimiri are with California Institute of Technology, Pasadena, California 91125 USA. 
The combination of many high-performance transistors and on-chip metal structures comparable to the wavelength, which can be used as on-chip radiators, is revolutionizing the design of millimeter-wave and submillimeter-wave transceivers and antennas.

In this article, the design of the millimeter-wave integrated antennas is discussed, the concept of nearfield direct antenna modulation (NFDAM) is introduced, and the implementation of a $60-\mathrm{GHz}$ proof-ofconcept chip based on NFDAM is presented.

\section{Millimeter-Wave Integrated Antennas}

One of the primary challenges in designing on-chip antennas on a silicon substrate is the high dielectric constant of silicon $\left(\varepsilon_{r}=11.7\right)$. To understand the importance of the dielectric constant, a simple dipole is placed on the boundary of semi-infinite regions of air and dielectric, as shown in Figure 1. The percentage of the power coupled into both the air and the dielectric is plotted in Figure 2. The figures indicate that for the case of a silicon substrate with $\varepsilon_{r}=11.7$, more than $95 \%$ of the power is radiated into the silicon rather than the air.

To avoid coupling the signal into the silicon substrate, a ground plane may appear to resolve the problem. There are two ways to implement this approach. One way is to make an on-chip ground plane by using

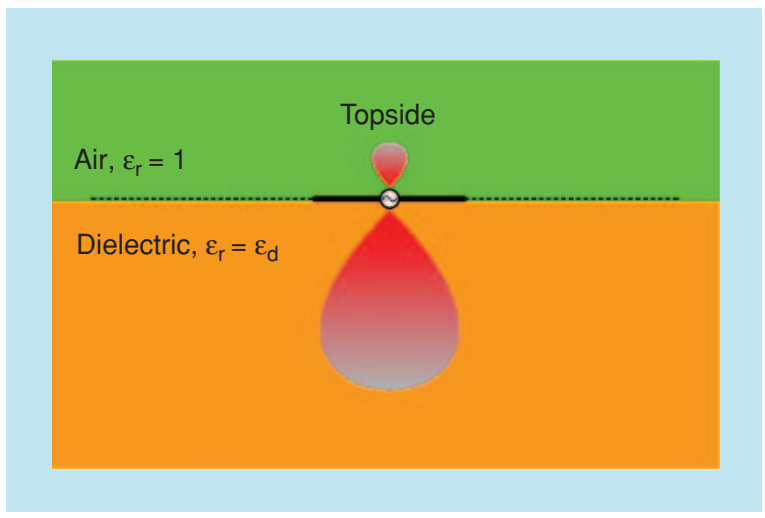

Figure 1. Radiating from top side without any ground shield [1]

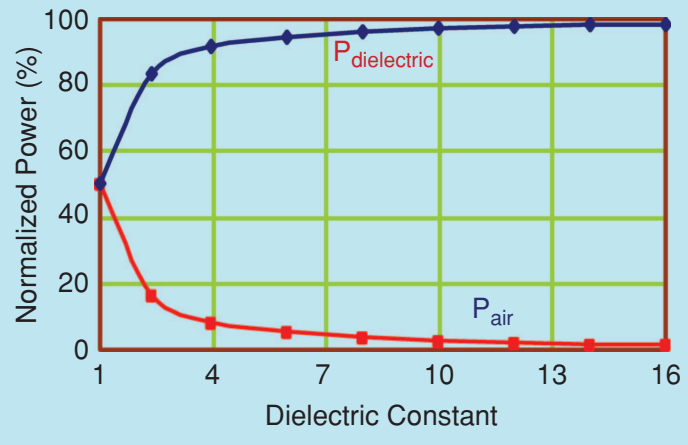

Figure 2. Ratio of the radiated power into air and into the dielectric substrate as a function of the dielectric constant [1].

\section{To understand the importance of the dielectric constant, a simple dipole is placed on the boundary of semi- infinite regions of air and dielectric.}

the lowest metal layer (e.g., $M_{1}$ ) while placing an onchip antenna on the topmost metal layer. Unfortunately, the distance between the topmost and the lowest metal layers is only a few micrometers in today's process technologies. This small distance almost shorts the antenna and reduces its efficiency to below 4-5\% [1]. The other way to implement a ground shield is to place an off-chip metal plane onto the backside of the silicon. In this case, because of the rectangular shape of the die and its thickness of several hundred micrometers, most of the dipole power gets coupled into substrate modes.

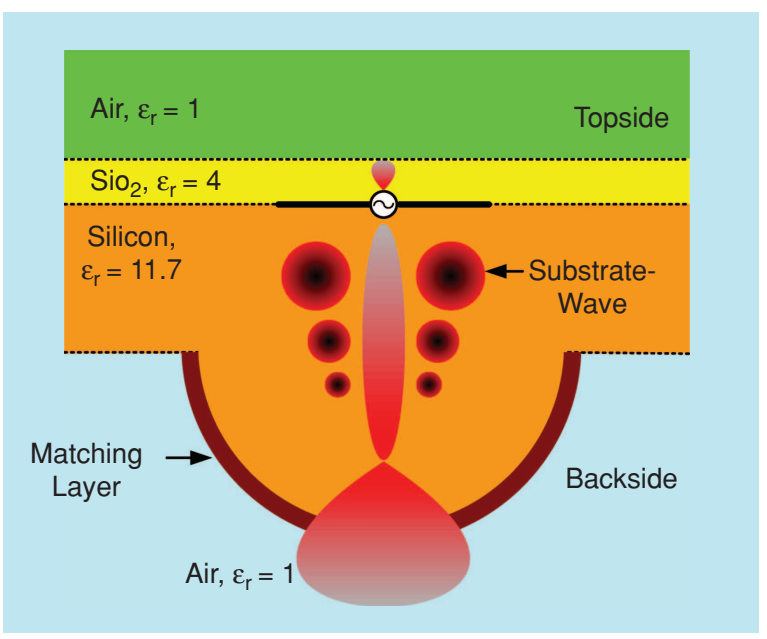

Figure 3. Radiating from the back side of the chip by means of a silicon lens [1].

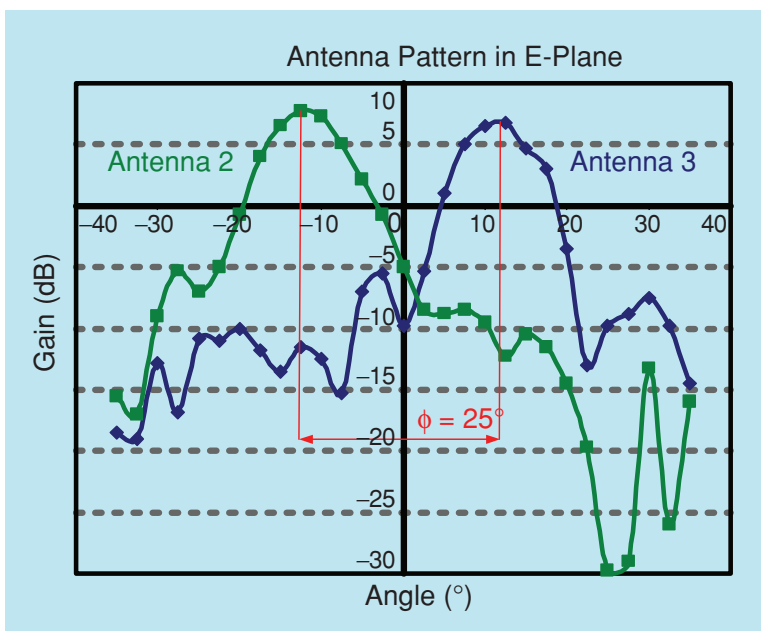

Figure 4. Measured E-plane patterns of two adjacent antennas on a silicon chip with a back side silicon lens. 


\section{In an NFDAM system, only a locked unmodulated carrier signal drives the antenna. In this case, the information is created by changing the phase and amplitude of the antenna pattern at different directions.}

A significant part of the power coupled into the substrate modes disappears as heat, and the rest is guided to the chip edge, which often results in an undesirable radiation pattern [1]. To avoid these problems and minimize the power coupled into substrate modes, a hemispherical dielectric lens, with a dielectric constant similar to that of silicon, can be placed on the back side of the silicon substrate [6]-[14]. The lens combines the substrate modes and converts them into useful radiated power, as illustrated in Figure 3.

To measure the antenna pattern, a W-band horn antenna is used to irradiate the on-chip dipoles on the receiver side. The E-plane patterns measured by the spherical method are shown in Figure 4. A maximum peak gain of $+8 \mathrm{dBi}$ is achieved in the measurement. Because of the off-axis properties of the silicon lens, the peak points of two patterns have an angular separation of $25^{\circ}$, which is close to the theoretical value of $23^{\circ}$ [14] corresponding to the lens dimensions shown in Figure 5.

\section{Near-Field Direct Antenna Modulation}

The availability of a large number of transistors with little incremental cost and high reproducibility in silicon enables a designer to take into account multiple levels of abstraction in a single chip-from system-, architecture-, circuit-, and device-level to electromagnetic structures. This interdisciplinary integration on multiple levels allows for implementing novel concepts and architectures that would not be feasible for conventional module-based approaches.

Near-field direct antenna modulation is a novel concept that is established by taking full advantage of such interdisciplinary integration capability [15], [16]. By combining antenna electromagnetic boundary conditions with data-transmitting architectures and circuits in a single integrated system, an efficient and secure communication scheme is possible, which is fundamentally different from conventional modulation schemes. The NFDAM-based system allows for direction-dependent data transmission with higher security than the conventional systems. In this section, the concept of the NFDAM technique is described in detail along with the associated advantages. Subsequently, the implementation of a NFDAM-based 60$\mathrm{GHz}$ integrated transmitter is described and accompanied by measurement

Figure 6. Modulation at baseband [16].

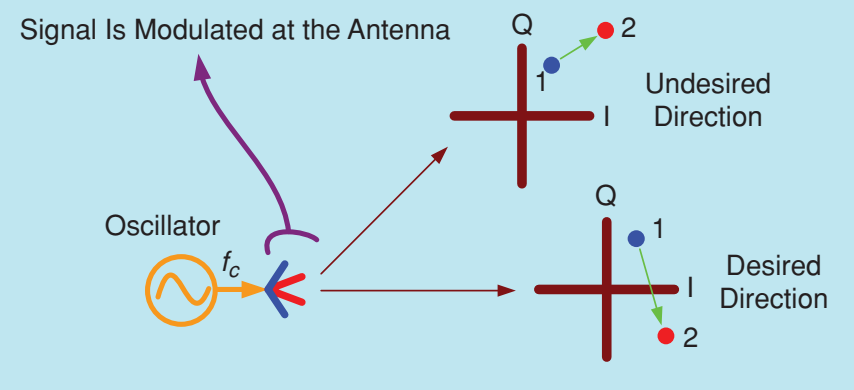

Figure 7. Modulation at antenna level [16]. results, which serve as a proof of concept.

To introduce the idea of NFDAM, two modulation schemes are illustrated in Figures 6 and 7. A conventional scheme is shown in Figure 6, where the information is created at baseband and the modulated signal is up-converted by a mixer. In this configuration, because the antenna pattern remains unchanged at each symbol transition, any change in the baseband signal appears at every direction in space. For example, if the amplitude of the baseband signal is increased by a factor of two, the amplitude of the transmitted signal will be doubled at every direction in space. In this case, an 


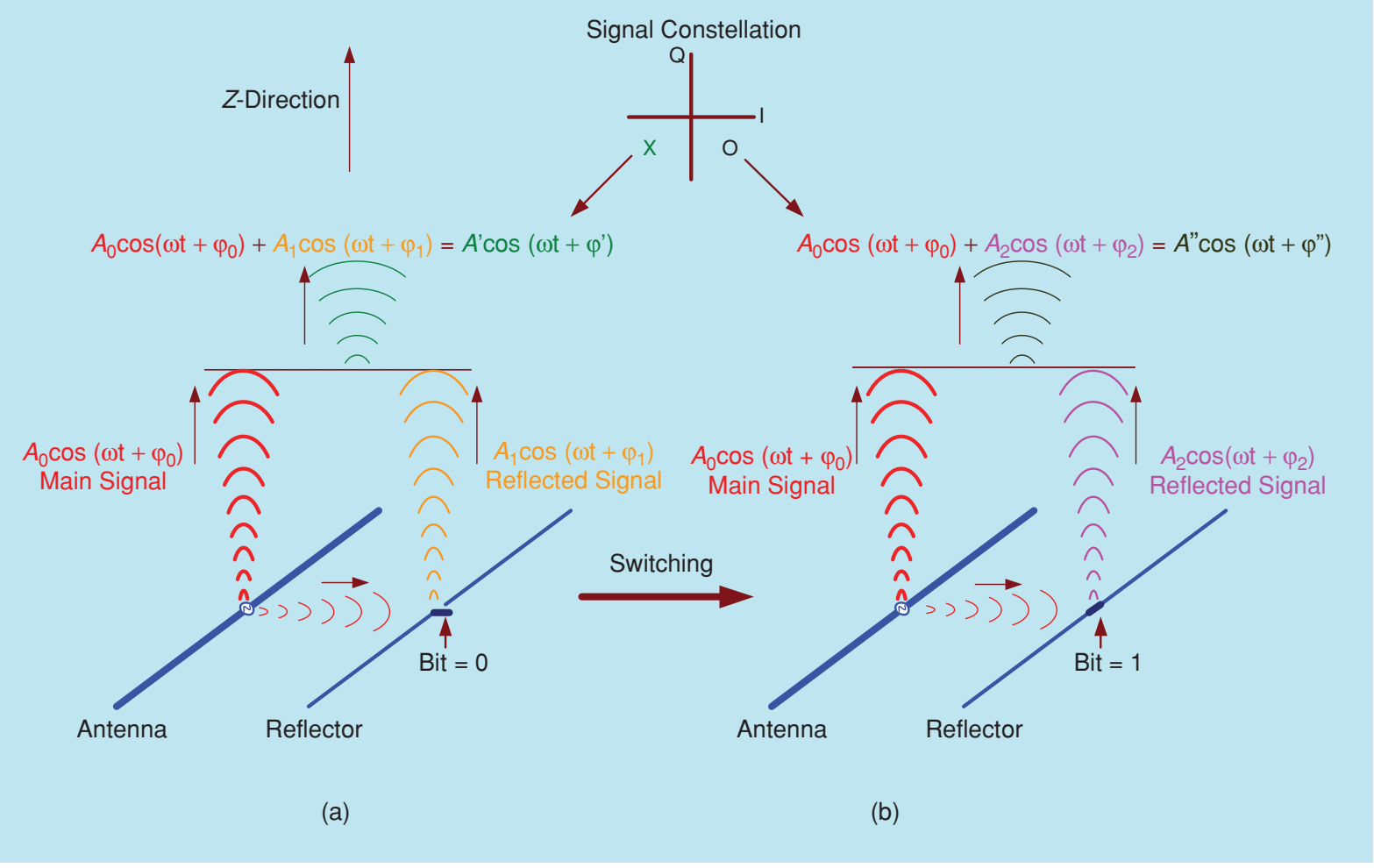

Figure 8. Signal modulation using switches on the reflectors (a) open switch and (b) closed switch [16].

eavesdropper with a sensitive receiver located at an undesired direction can receive all the changes in the baseband data and hence properly demodulate the signal. In an NFDAM system, only a locked unmodulated carrier signal drives the antenna. In this case, the information is created by changing the phase and amplitude of the antenna pattern at different directions. In other words, because the antenna pattern changes at each symbol transmission, the change in amplitude and phase of the received signal becomes a function of the direction. In this case, it is possible to double the amplitude of the received signal at one direction but decrease it (or leave it unchanged) in another direction. Figure 7 illustrates the idea of NFDAM, where the information is sent to the desired direction and the misinformation is sent in the undesired directions. In an NFDAM system, in order to modulate the signal, the antenna

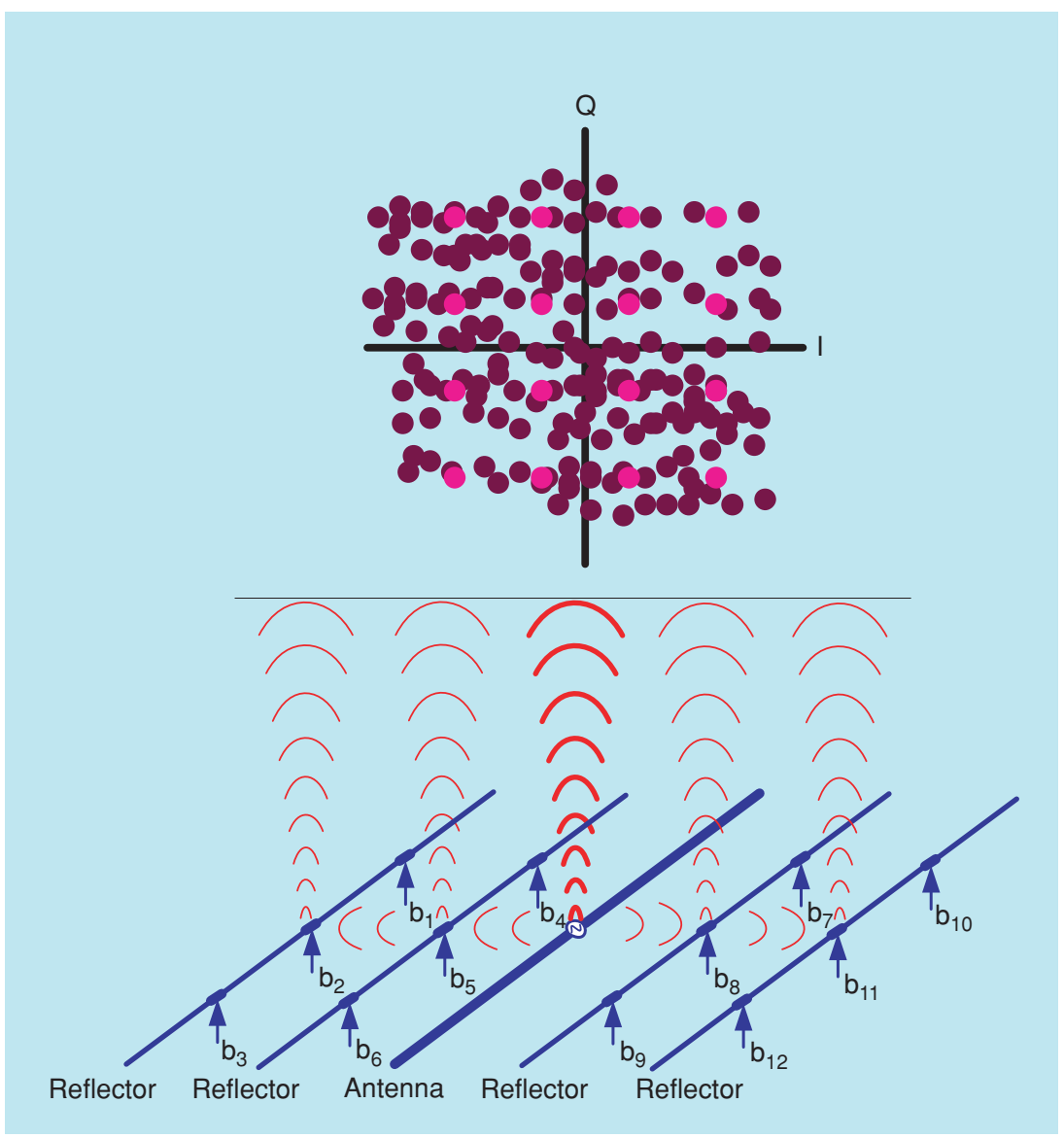

Figure 9. Arbitrary signal modulation [16]. 


\section{At the undesired direction, constellation points are completely scrambled demonstrating the functionality of the NFDAM system.}

characteristics (its near- and far-field) have to vary at the symbol rate.

An oversimplified example of the NFDAM technique is shown in Figure 8, where a reflector is placed in close proximity to a dipole antenna. An ideal switch is placed in the middle of this reflector to change its

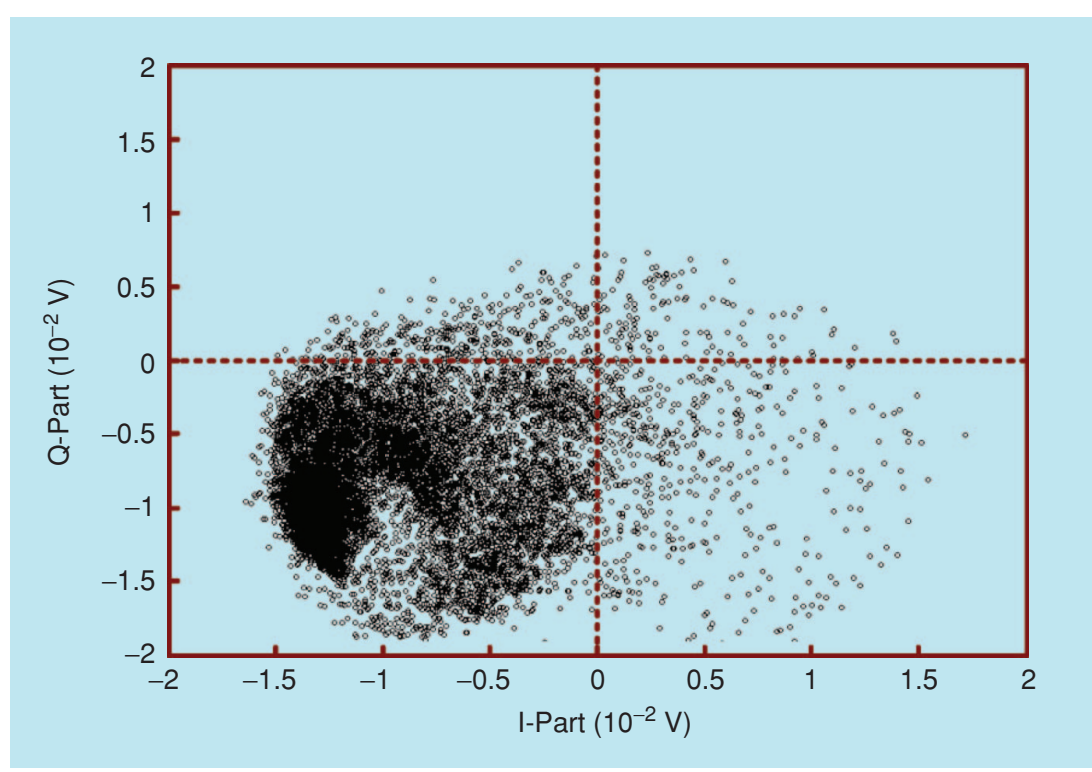

Figure 10. Simulation results of the switch-based NFDAM transmitter (10,000 points). effective electrical length. Let us assume the main dipole antenna radiates a signal $A_{0} \cos \left(\omega t+\varphi_{0}\right)$ in the $z$-direction, normal to the plane (boresight). Some part of the main signal couples to the adjacent reflector in the near field of the antenna, causing the reflector to scatter a signal $A_{1} \cos \left(\omega t+\varphi_{1}\right)$ at its open state and a signal $A_{2} \cos \left(\omega t+\varphi_{2}\right)$ at its closed state. On the basis of this assumption, the far-field signal in the $z$-direction can be calculated by

$$
\text { Open switch: } A_{0} \cos \left(\omega t+\varphi_{0}\right)+A_{1} \cos \left(\omega t+\varphi_{1}\right)
$$$$
=A^{\prime} \cos \left(\omega t+\varphi^{\prime}\right) \text {. }
$$

Closed switch: $A_{0} \cos \left(\omega t+\varphi_{0}\right)+A_{2} \cos \left(\omega t+\varphi_{2}\right)$

$$
=A^{\prime \prime} \cos \left(\omega t+\varphi^{\prime \prime}\right) \text {. }
$$

Figure 8 shows the real and imaginary parts of the combined signal in the far field. Although this oversimplified example shows how a crude signal modulation can be done by a simple switch, it does not capture the complete near-field electromagnetic behavior of the system. In our design, where reflectors are placed in the near field of the antenna, changes in the antenna parasitic components modulate the far-field radiated signal without necessarily varying the path delay.

Figure 9 shows a more practical configuration where a total number of $N$ switches produce $2^{N}$ constellation points. By having a sufficiently large number of switches, it is possible to cover most of

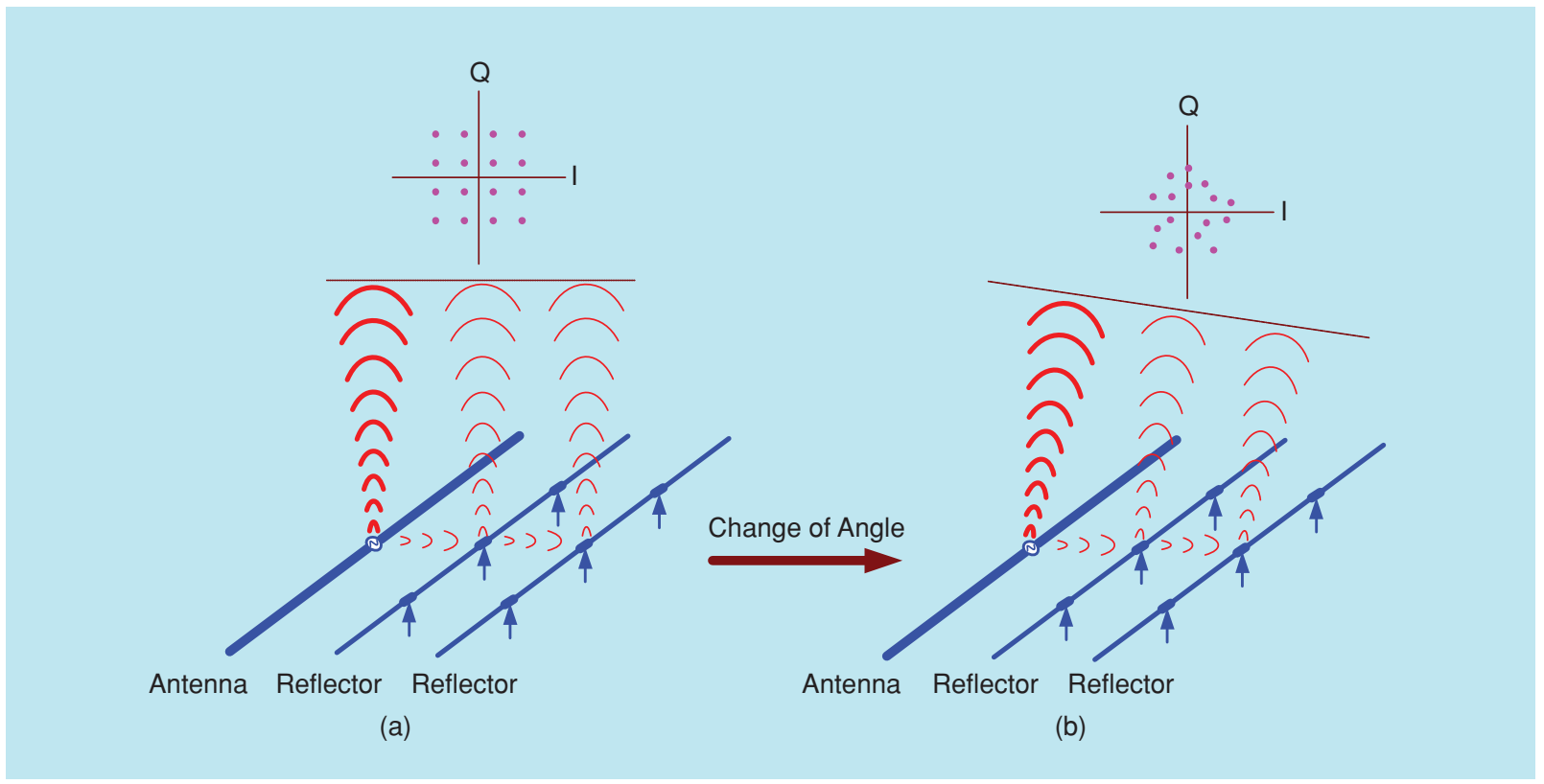

Figure 11. Communication security: (a) desired direction and (b) undesired direction [16]. 

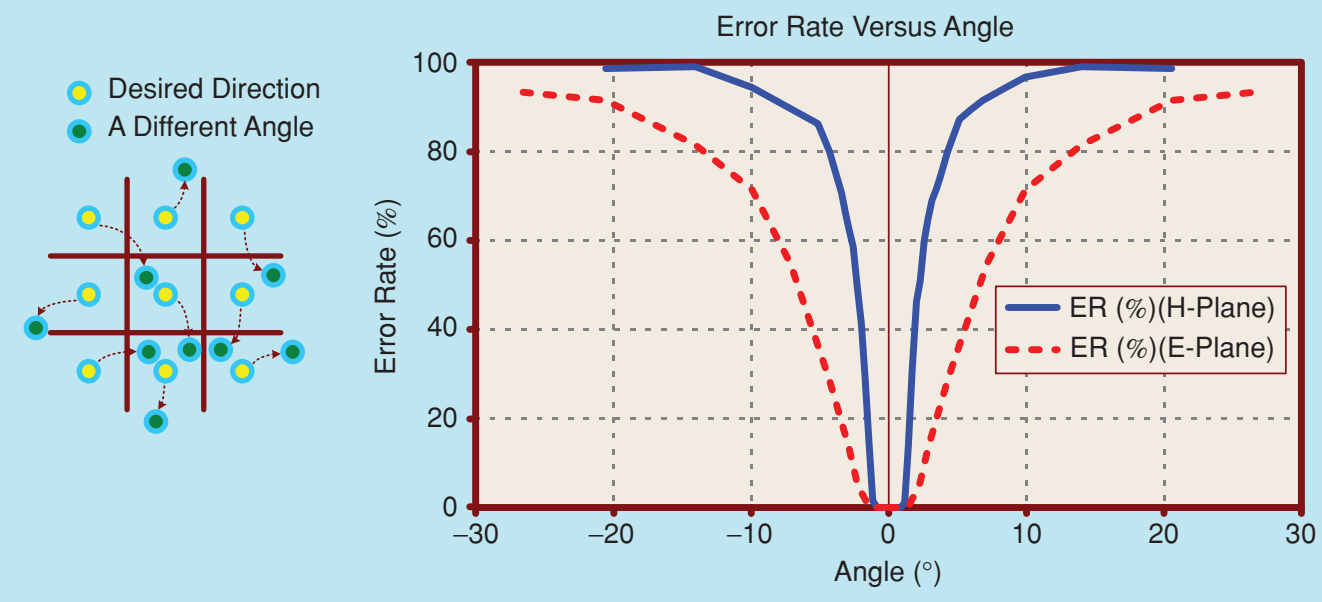

Figure 12. Information beamwidth for a modulation with 210 equally spaced constellation points [16].

the points on the signal constellation diagram and select any arbitrary modulation (Figure 9). Figure 10 shows a simulation result where 10,000 random switching combinations generate 10,000 distinct points on the signal constellation diagram. Each point corresponds to an induced voltage on a receiving dipole antenna located at the far field. In this simulation, five reflectors are placed at each side of the antenna and nine ideal switches are placed on each reflector, resulting in a total number of 90 switches.

By changing the switching configuration, the near field of the antenna and hence its impedance are changed, but this can be alleviated by exploiting the redundancy. For a maximum of $1.5 \mathrm{~dB}$ variation in power gain, $2 \mathrm{dBm}$ variation in delivered power, and $3 \%$ variation in power added efficiency of the power amplifier (PA), it is possible to cover one quadrant with resonant switches that present a maximum impedance (off impedance) of $70 \Omega$.

\section{Properties of Systems Utilizing Near-Field Direct Antenna Modulation}

\section{Using a Narrowband (PA) to Transmit Wideband Information}

In NFDAM systems, because the modulated signal does not pass the PA, specifications/requirements on the PA and the modulated signal can be totally different. In fact, a highly efficient narrowband switching PA can be used to amplify the carrier signal while antenna parasitic modulation can be utilized to transmit a nonconstant-envelope wideband signal.

\section{Secure Communication Link and Information Beam Width}

Assume that a set of switching combinations can be found that generates 16 quadrature amplitude modulation (QAM) points at boresight [Figure 11(a)]. If the

\section{A highly efficient narrowband switching PA can be used to amplify the carrier signal while antenna parasitic modulation can be utilized to transmit a nonconstant-envelope wideband signal.}

receiver moves to a different direction, it will see scrambled points on the signal constellation diagram [Figure 11(b)]. This is because the scattering properties of these reflectors-and hence the phase and amplitude of the reflected signal-vary with angle. This is one of the unique properties of the NFDAM systems that prevent undesired receivers from properly demodulating the signal. Figure 12 shows that the constellation points start to move to seemingly random locations, and this movement is increased at larger angles. As Figure 12 depicts, some of these points fall into adjacent compartments and introduce error. The simulated error rate versus angle in the E-plane (parallel to the dipole) and the $H$-plane (normal to the dipole) of an on-chip dipole antenna is also shown in

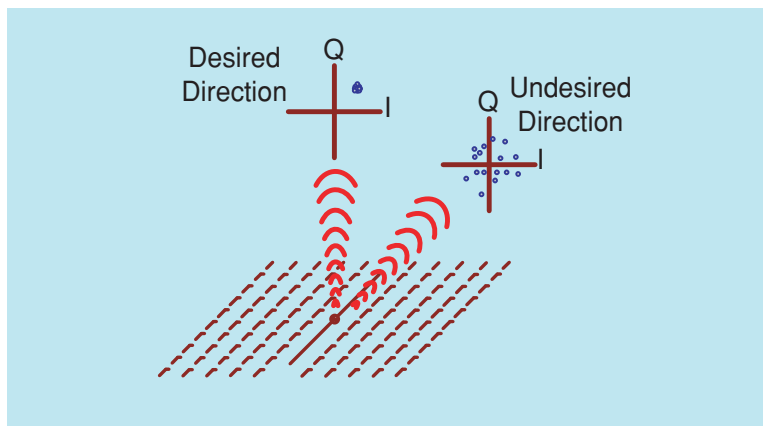

Figure 13. Enhancing security by leveraging redundancy [16]. 


\section{NFDAM systems provide a unique solution for transmitting highly secured direction-dependent communication data.}

Figure 12, where 210 equally spaced constellation points at boresight are selected and viewed at different angles. In the $H$-plane, the error rate rises rapidly and

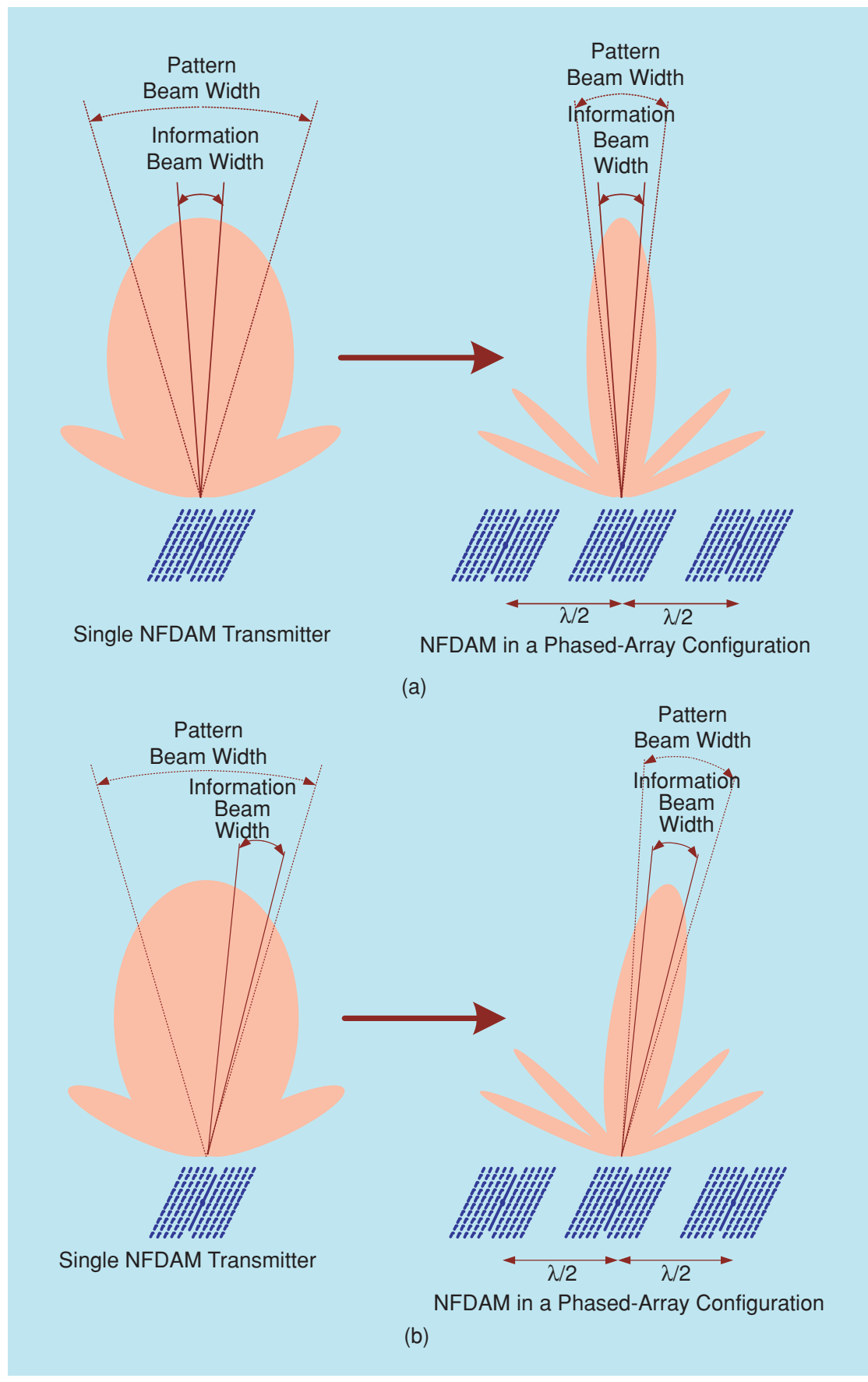

Figure 14. NFDAM transmitter in a phased-array configuration: (a) transmitting to boresight and (b) transmitting to a different angle [16]. reaches approximately $50 \%$ at offset angles of 2 to 3 degrees. In the E-plane, the error rate reaches approximately $50 \%$ at offset angles of 6 to 7 degrees off boresight. Receivers located at angles $\pm 1^{\circ}$ can completely recover the modulated signal in the absence of noise and other channel nonidealities. From this simulation, the information beamwidth of the antenna, which is a metric of information directionality of the beam, can be defined as the angle in which a noiseless receiver can recover the signal without introducing any error. For NFDAM systems, the information beamwidth represents the information directionality of the beam while the power beamwidth represents the power directionality of the antenna pattern.

\section{Redundancy and Added Security}

With enough redundancy, it is possible to find different switch combinations that produce almost the same point in the desired direction while generating widely scattered points in other directions (Figure 13). This unique property of NFDAM systems makes it possible to transmit a set of predefined modulation points in a desired direction while simultaneously randomly changing the pattern of the constellation points in the undesired directions, preventing unintended receivers from finding a one-to-one mapping between the received signal at the desired direction and the undesired directions.

\section{Phased-Array Configuration}

Because direct antenna modulation is performed in the near field of the antenna, each NFDAM system can behave as a single element in a phasedarray configuration. In this case, NFDAM system can control the information beamwidth of the antenna while the phased-array functionality can be used to narrow the power beamwidth of the array pattern and hence increase the power efficiency of the whole system, as illustrated in Figure 14. 


\section{Implementing a NFDAM 60-GHz Transmitter}

Figure 15 shows the block diagram of the $60-\mathrm{GHz}$ transmitter [15], [16], which is implemented in the IBM 130-nm BiCMOS Si-Ge (8HP) process. In this design, five reflectors are placed on each side of the antenna and nine switches are placed on each reflector, resulting in a total of 90 switches. Reflectors are implemented on metal layers $M_{1}$ to $M_{3}$, each having a length of $1,300 \mu \mathrm{m}$. A dipole antenna with a length of $835 \mu \mathrm{m}$ is also implemented on metal layers $M_{1}$ to $M_{3}$. A differential carrier signal generated by an on-chip locked voltage-controlled oscillator (VCO) is amplified with an on-chip PA. This signal travels inside a shielded differential transmission line implemented on top metal layers $M_{6}$ and $M_{7}$. As shown in Figure 15, the baseband data control the state of switches through a digital control unit. The electromagnetic structure, including the dipole antenna and reflectors, occupies an area of $1.3 \times 1.5 \mathrm{~mm}^{2}$.

The coverage of the signal constellation diagram is a function of size, shape, and location of the reflectors as well as their distance from the main antenna. If the reflectors are placed very close to the antenna, they will not be able to generate a sufficient number of radiating modes necessary to transmit independent signals to multiple directions simultaneously. On the other hand, if the reflectors are placed too far from the main antenna, the reflected signal will be very weak and hence will not be able to significantly affect the phase and amplitude of the main signal. In this design, in order to maximize the coverage of the signal constellation diagram, the distance of the reflectors from the main antenna is optimized.

Figure 16 shows the block diagram of an optional coarse control unit that can behave as a quadrant selector with control signals $A$ and $B$ taking values 1 and -1 . This unit can be turned on in situations where the antenna parasitic modulation fails to generate widely scattered points to cover the four quadrants.
The coverage of the signal constellation diagram is a function of size, shape, and location of the reflectors as well as their distance from the main antenna.

One of the challenging tasks of the design is implementing switches with a relatively high ratio of off and on impedances. The switch size cannot be very small as it has to provide a small impedance (about 1 to $5 \Omega$ ) at its closed state. On the hand, a large switch limits the maximum impedance achievable at the open state. To

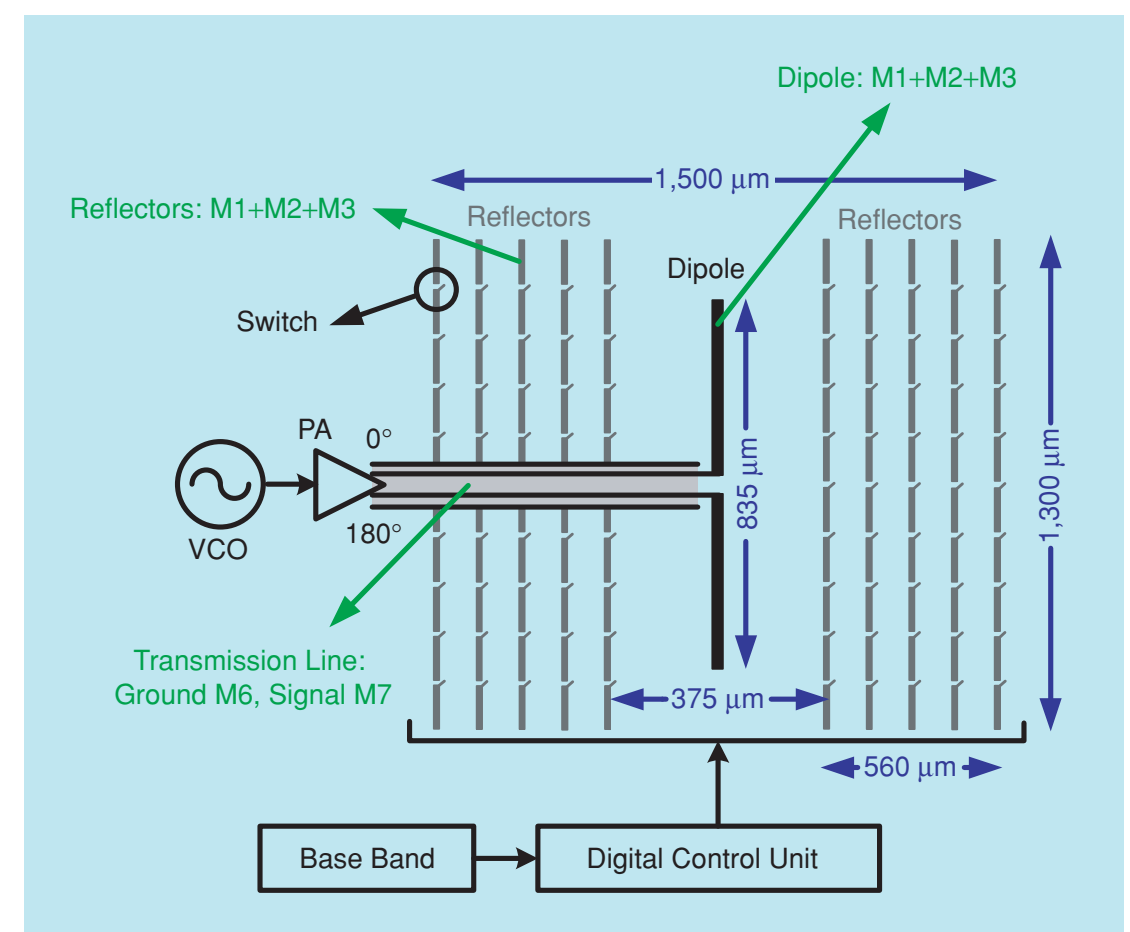

Figure 15. Block diagram of the NFDAM transmitter architecture [16].

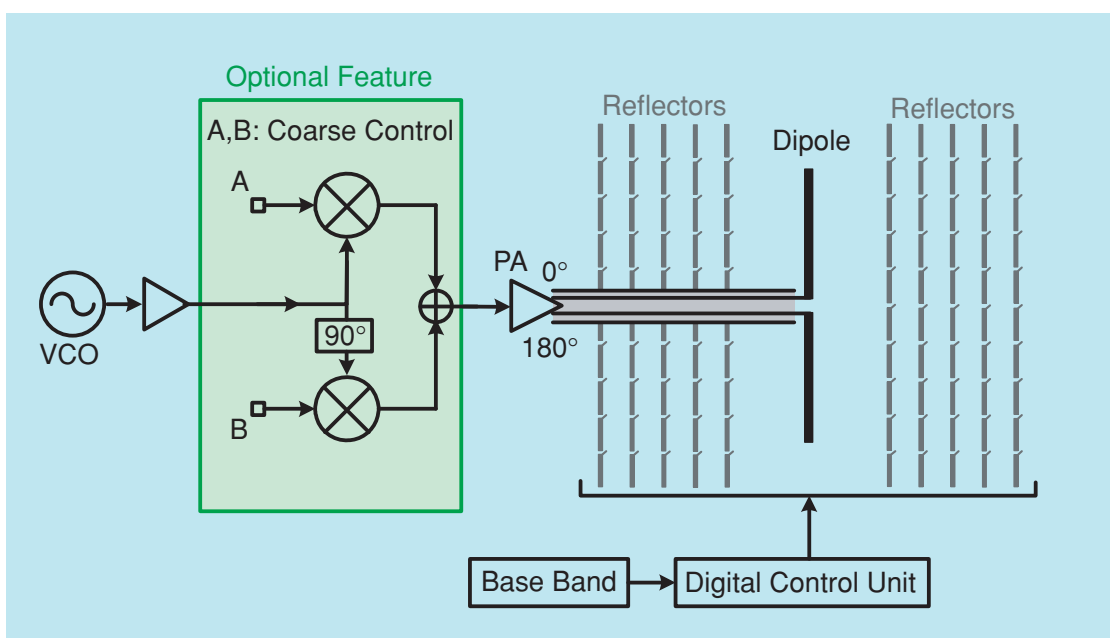

Figure 16. Optional coarse control unit (quadrant selector) [16]. 
alleviate this problem, a circular shielded transmission line is used to connect the drain and source of the Ntype metal-oxide-semiconductor (NMOS) switch res-

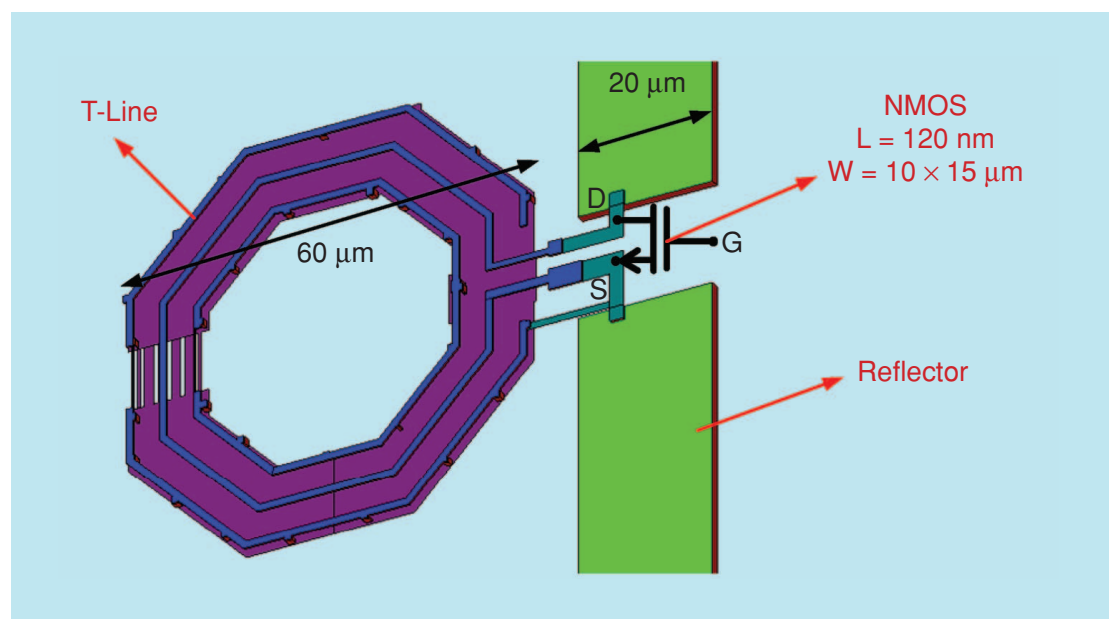

Figure 17. A 60-GHz resonant NMOS switch [16].

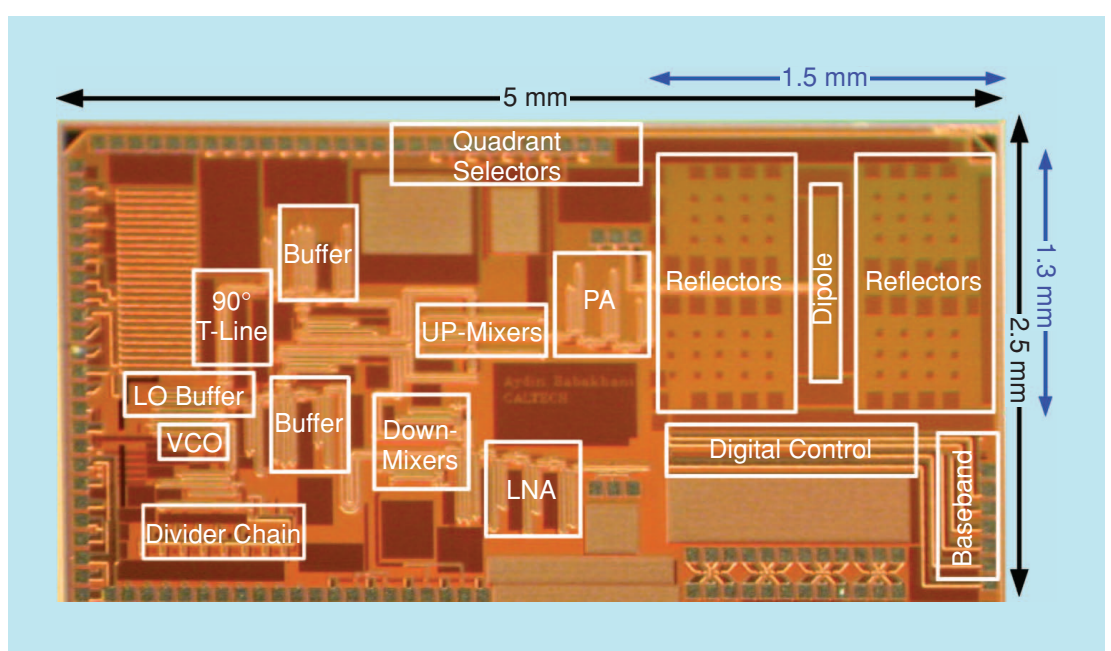

Figure 18. NFDAM chip micrograph [16].

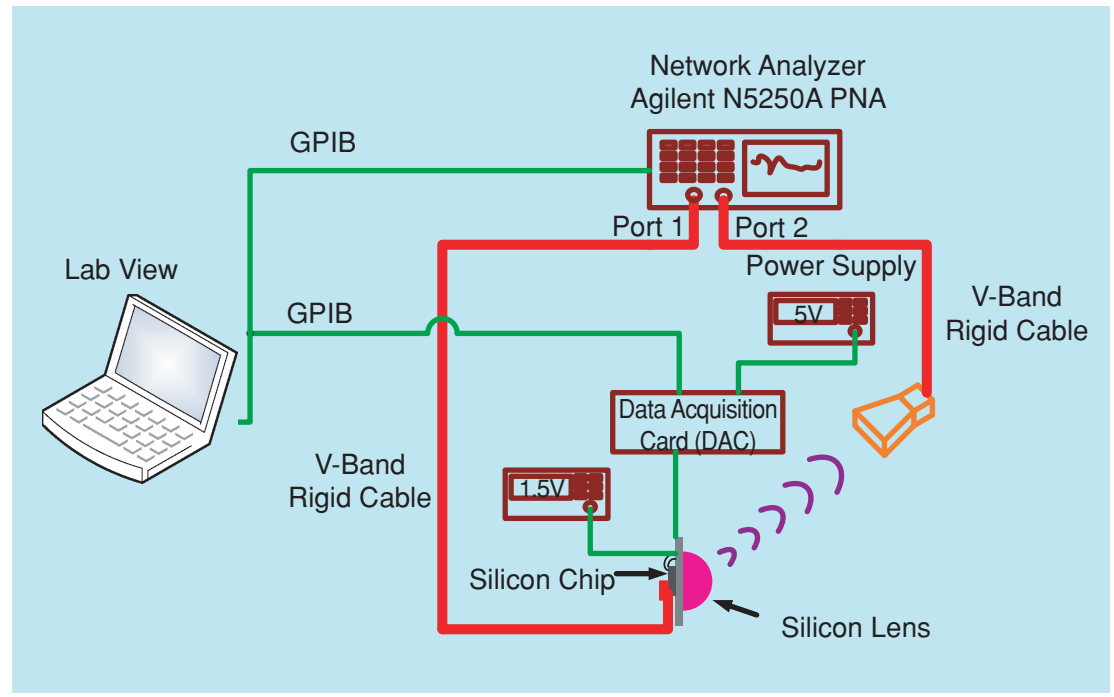

Figure 19. Measurement setup [16]. onating out the parasitic capacitance of the switch in its open state and hence increasing the off impedance of the switch (Figure 17). Utilizing this technique provides a maximum off impedance of $70 \Omega$ at 60 GHz. The NMOS switch used in this design has a length of $120 \mathrm{~nm}$ and a width of $150 \mu \mathrm{m}$. The circular transmission line is implemented on metal layers $M_{2}$ to $M_{4}$ and has a diameter of $60 \mu \mathrm{m}$.

A micrograph of the chip is shown in Figure 18, which includes the reflectors, dipole antenna, PA, up-converter mixers, local oscillator (LO) buffers, VCO, injection locked divider, digital divider chain, and baseband buffers. In addition to the transmitter, a complete receiver including low-noise amplifier (LNA), down-converter mixers, baseband buffers, and receiving on-chip antennas has been designed and implemented.

\section{Measurement Results}

A hemispherical silicon lens with a diameter of $25.4 \mathrm{~mm}$ and an extension length of $1 \mathrm{~mm}$ is placed on the back side of the silicon chip. This lens minimizes the substrate modes by converting them into useful radiation modes, as described above. Figure 19 shows the block diagram of the measurement setup. A LabView program communicates with a data acquisition card through general purpose interface bus (GPIB) ports. The data acquisition card transmits the baseband data to an on-chip digital control unit and controls the state of switches. The LabView program also communicates with an Agilent N5250A PNA network analyzer using a GPIB card. The network analyzer measures the real and imaginary parts of $S_{21}$ for every switching combination. In this measurement, the transmitter is disconnected (with laser trimming) from the on-chip dipole antenna. In fact, a V-band signal generated by a network analyzer directly drives the on-chip dipole antenna through a wire-bond connection. The other port of the network analyzer measures the received signal at the horn antenna.

Figure 20 shows the measured real and imaginary parts of the $S_{21}$ in two different directions with an angular separation of approximately $90^{\circ}$. In this measurement, the same set of switching combinations is used for both directions. Out of 2,000 switching combinations, which are selected 
randomly, 20 equally spaced constellation points in the desired direction are chosen. For the same set of switching combinations, real and imaginary parts of $S_{21}$ are measured at an undesired direction as shown in Figure 20. At the undesired direction, constellation points are completely scrambled demonstrating the functionality of the NFDAM system. Vertical color coding is used to emphasize the scrambling effect.

In another measurement, the optional quadrantselector unit in conjunction with reflector switching is
For NFDAM systems, the information beamwidth represents the information directionality of the beam while the power beamwidth represents the power directionality of the antenna pattern.
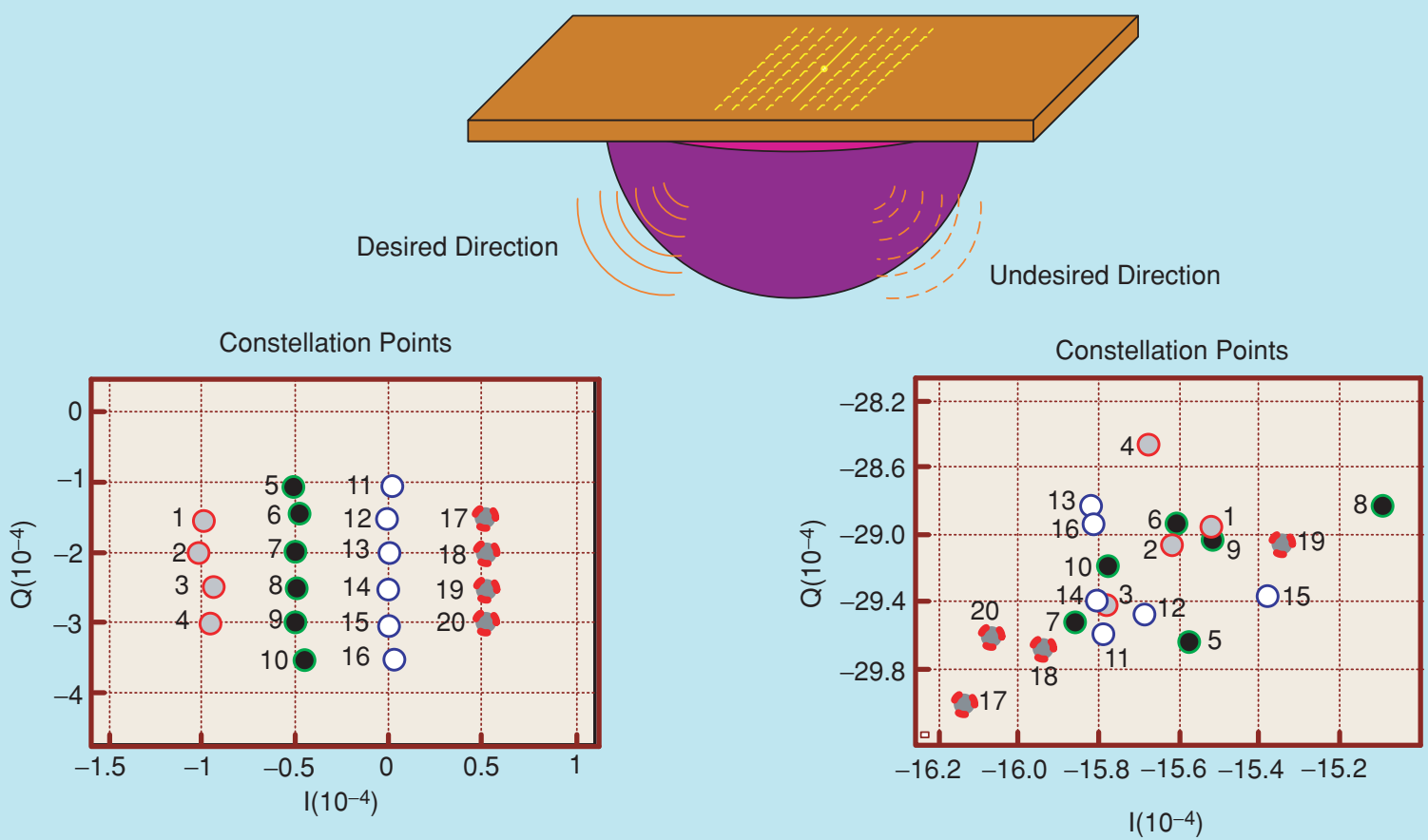

Figure 20. Measured constellation points of the switch-based NFDAM chip. In this measurement only the switches are used [16].

used to cover the four quadrants on the signal constellation diagram (Figure 21). The picture of the measurement setup is shown in Figure 22.

To verify the repeatability of $S$-parameter measurement, several methods are used. In one case, real and imaginary parts of $S_{21}$ are measured for a fixed set of switching combinations 1000 times. This measurement was intentionally performed over the course of 10 hours to capture the supply voltage noise, room temperature change, and other variations. According to the measured results, the error in repeatability is much smaller than the distance of two adjacent points in Figures 20 and 21.

A separate measurement is made to characterize the performance of the transmitter. In this measurement, the antenna is disconnected from the PA (with laser trimming) and a V-band wafer probe is used to capture the output power of the

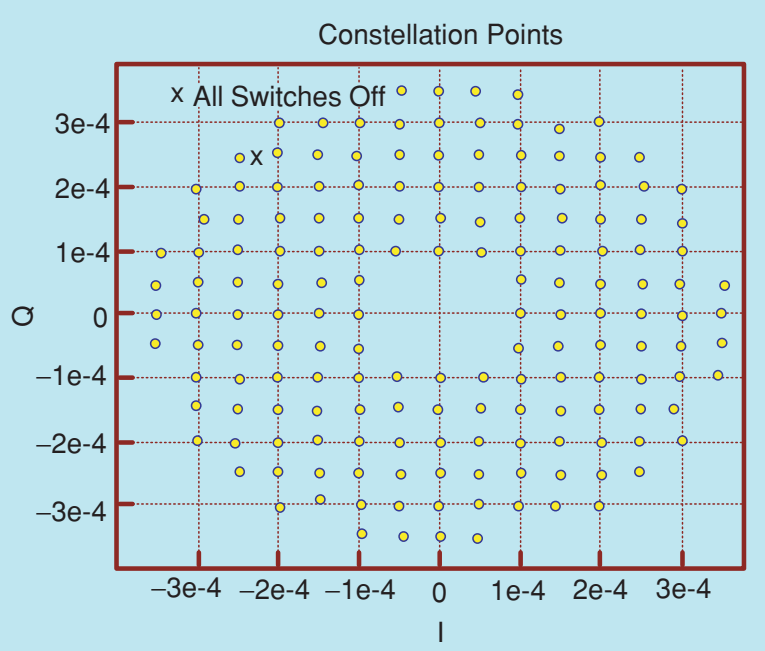

Figure 21. Measurement results of the four-quadrant coverage of the signal constellation space using the optional quadrant-selector and switches (switch-based NFDAM chip). 


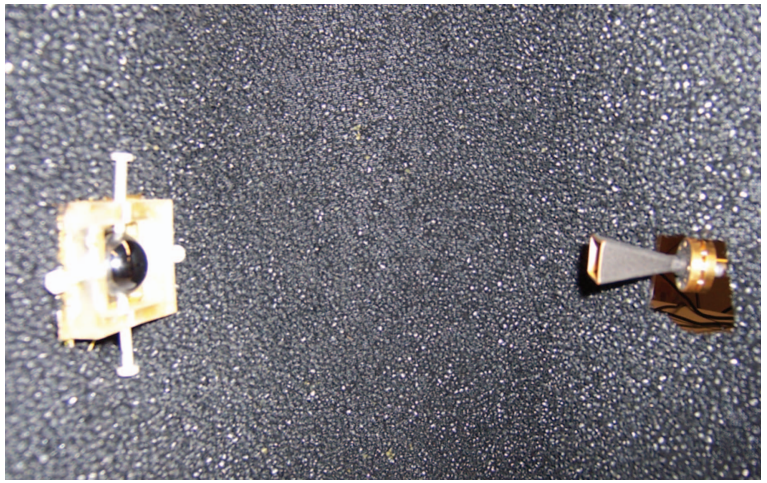

Figure 22. Measurement setup.

\section{NFDAM systems provide a unique solution for transmitting highly secured direction-dependent data and hence preventing eavesdroppers from properly demodulating the signal.}

transmitter. An Agilent signal source (E8257D) sends the input signal to the transmitter input. An Agilent Vband power sensor (V8684A) and power meter (E4418B) are used to measure the output power of the transmitter. The measured results indicate an output power of $+7 \mathrm{dBm}$, a small-signal gain of $33 \mathrm{~dB}$, and a saturated gain of $25 \mathrm{~dB}$.

In a separate measurement, VCO performance is characterized by directly connecting the on-chip probes to the output of an LO buffer. Phase noise and tuning range of the $\mathrm{VCO}$ are measured by an Agilent spectrum analyzer (E4448A) and a SpacekLabs off-chip down-converter (GE-590). This measurement shows a tuning range of more than $2.5 \mathrm{GHz}$ and a phase noise of $-100 \mathrm{dBc}$ at $10 \mathrm{MHz}$ offset.

\section{Conclusions}

NFDAM systems provide a unique solution for transmitting highly secured direction-dependent data and hence preventing eavesdroppers from properly demodulating the signal. A $60-\mathrm{GHz}$ proof-of-concept chip was designed and measured.

\section{Acknowledgments}

The authors appreciate the help of Yu-Jiu Wang of the California Institute of Technology and support of the Lee Center for Advanced Networking at
California Institute of Technology. The technical support from Cadence Design Systems for CAD tools and from Agilent Technologies, Zeland Software Inc., and Ansoft Corporation is also appreciated.

\section{References}

[1] A. Babakhani, X. Guan, A. Komijani, A. Natarajan, and A. Hajimiri, "A $77 \mathrm{GHz}$ phased array transceiver with on chip dipole antennas: Receiver and on-chip antennas," IEEE J. Solid-State Circuits, vol. 41, no. 12, pp. 2795-2806, Dec. 2006.

[2] A. Natarajan, A. Komijani, X. Guan, A. Babakhani, and A. Hajimiri, "A $77 \mathrm{GHz}$ phased array transmitter with local LO-path phaseshifting in silicon," IEEE J. Solid-State Circuits, vol. 41, no. 12, pp. 2807-2819, Dec. 2006.

[3] B.A. Floyd, S.K. Reynolds, U.R. Pfeiffer, T. Zwick, T. Beukema, and B. Gaucher, "SiGe bipolar transceiver circuits operating at $60 \mathrm{GHz}$," IEEE J. Solid-State Circuits, vol. 40, no. 1, pp. 156-167, Jan. 2005.

[4] B. Razavi, "A 60-GHz CMOS receiver front-end," IEEE J. SolidState Circuits, vol. 41, no. 1, pp. 17-22, Jan. 2005.

[5] C.H. Doan, S. Emami, A.M. Niknejad, and R.W. Brodersen, "Millimeter-wave CMOS design," IEEE J. Solid-State Circuits, vol. 40, no. 1, pp. 144-155, Jan. 2005.

[6] D.B. Rutledge, D.P. Neikirk, and D. Kasilingam, "Integrated-circuit antennas," in Infrared and Millimeter-Waves. New York: Academic, 1983, pp. 1-90.

[7] N. Engheta and C.H. Papas, "Radiation patterns of interfacial dipole antennas," Radio Sci., vol. 17, no. 6, pp. 1557-1566, 1982.

[8] H. Kogelnik, "Theory of dielectric waveguides," in Integrated Optics, T. Tamir, Ed. New York: Springer-Verlag, 1979, ch. 2, pp. 66-79.

[9] N.G. Alexopoulos, P.B. Katehi, and D.B. Rutledge, "Substrate optimization for integrated circuit antennas," IEEE Trans. Microwave Theory Tech., vol. 83, no. 7, pp. 550-557, July 1983.

[10] B. Chantraine-Barès, R. Sauleau, L. Le Coq, and K. Mahdjoubi, "A new accurate design method for millimeter-wave homogeneous dielectric substrate lens antennas of arbitrary shape," IEEE Trans. Antennas Propagat., vol. 53, no. 3, pp. 1069-1082, Mar. 2005.

[11] T. Nagatsuma, A. Hirata, M. Harada, H. Ishii, K. Machida, T. Minotani, H. Ito, T. Kosugi, and T. Shibata, "Millimeter-wave photonic integrated circuit technologies for high-speed wireless communications applications," in IEEE ISSCC Dig. Tech. Papers, Feb. 2004, pp. 448-449.

[12] M.J.M. van derVorst, P.J.I. de Maagt, and M.H.A.J. Herben, "Effect of internal reflections on the radiation properties and input admittance of integrated lens antennas," IEEE Trans. Microwave Theory Tech., vol. 47, no. 9, pp. 1696-1704, Sept. 1999.

[13] P. Focardi, W.R. McGrath, and A. Neto, "Design guidelines for terahertz mixers and detectors," IEEE Trans. Microwave Theory Tech., vol. 53, no. 5, pp. 1653-1661, May 2005.

[14] D.F. Filipovic, G.P. Gauthier, S. Raman, and G.M. Rebeiz, "Off axis properties of silicon and quartz dielectric lens antennas," IEEE Trans. Antennas Propagat., vol. 45, no. 5, pp. 760-766, May 1997.

[15] A. Babakhani, D.B. Rutledge, and A. Hajimiri, "A near-field modulation technique using antenna reflector switching," in IEEE ISSCC Dig. Tech. Papers, Feb. 2008, pp. 188-189.

[16] A. Babakhani, D.B. Rutledge, and A. Hajimiri, "Transmitter architectures based on near-field direct antenna modulation," IEEE J. Solid-State Circuits, vol. 43, no. 12, Dec. 2008. 